\title{
A Proposal for Continuous Evaluation-based Interactive Evolutionary Computation
}

\author{
Makoto Fukumoto \\ Faculty of Information Engineering, Fukuoka Institute of Technology \\ 3-30-1 Wajiro-higashi, Higashi-ku, Fukuoka, 811-0295 Japan. \\ E-mail: fukumoto@fit.ac.jp \\ Yoshiko Hanada \\ Faculty of Engineering Science, Kansai University \\ 3-3-35 Yamate-cho, Suita-shi, Osaka, 564-8680 Japan. \\ E-mail: hanada@kansai-u.ac.jp
}

\begin{abstract}
Interactive Evolutionary Computation (IEC) is known as an efficient method which reflects user's subjective preference on media contents. However, user's fatigue caused by repetitive evaluation tasks remains as a severe problem in IEC. The purpose of this study is to propose a new framework of IEC, continuous evaluation-based IEC (CEIEC) that continues user's evaluation tasks in many days. CEIEC allows the user's subjective evaluation day by day to have many evaluation times without severe fatigue, while conventional IECs performed user's task in one time in one day. This study also performed a listening experiment for investigating efficiencies of CEIEC. The target of search with CEIEC was to design a bright sign sound. Sixteen subjects participated in the listening experiment which is composed of tasks of three days: in the 1 st and the 2nd days, the subjects performed a selection task throughout fifteen generations. Successive update of the solution was observed during these two days. In the 3rd day, the subjects evaluated three sign sounds with 7-point scale. These three sounds were picked up from the 0th, 14th, and 29th generations, respectively. The sound with the highest score was found in the 29th generation. These results show the efficiencies of CEIEC.
\end{abstract}

\section{Introduction}

Using media contents suited to our feelings is important to make our life enrich. Recent development of information technologies enables us to provide media contents suited to feelings of each of us. The ability of these technologies is still limited, because of the user's feeling is different between the users and is a kind of black box that is difficult to represent by users themselves.

Interactive Evolutionary Computation (IEC) [1,2] is known as an efficient method which reflects user's subjective preference on the contents. IEC employs user's subjective evaluation for evaluation process in Evolutionary Computation (EC), which is well-known stochastic searching method of optimal solutions in various problems.
IEC was mainly applied for media contents related to computer graphics and sounds [2], and recent IEC studies have developed a new method related to vibration [3-6] and fragrance $[7,8]$.

However, user's mental fatigue remains as a serious problem in IEC. The fatigue is caused by evaluation tasks in IEC where the users have to subjectively evaluate solution candidates many times. Furthermore, because of the user's fatigue, evaluation time is limited in IEC. According to Takagi's survey [2], most of conventional IEC studies performed from 10 to 20 generations as user's task. The number of evaluation times is directly related to searching ability of EC, therefore, the limitation of evaluation time is a weak point that prevents finding better and optimal solutions.

The purpose of this study is to propose a new framework of IEC that continues user's evaluation tasks in many days: we name it continuous evaluation-based IEC (CEIEC). The proposed IEC allows the user's subjective evaluation day by day to have many evaluation times without severe fatigue, while conventional IECs performed user's task in one time in one day. It seems natural that keeping evaluation times day by day dedicates to find better and/or the best solutions in IEC. However, it is not clear whether the user's feeling is same or not every day, and it has not been investigated in previous studies. This study also performed an experiment for investigating efficiencies of the proposed IEC.

\section{Continuous Evaluation-based Interactive Evolutionary Computation}

Conventional IEC enables an individual user to obtain media contents suited to each user's feelings through stochastic search. However, user's fatigue caused by the repetitive evaluation task on media contents, solution candidates of EC, was a severe problem in IEC [2]. Fig. 1 shows a fundamental scheme of IEC that searches optimal or better solutions suited to user's feelings. The EC system presents solution candidates as stimuli to the user, and the user subjectively evaluates them by procedure of scoring or 
selection. The user's task in conventional IECs is performed in one time in one day, therefore, the evaluation time is limited by user's fatigue.

The basic idea of the proposed method, CEIEC, is to divide the evaluation task into different times in different days. It means that result of search in IEC in the 1 st day is taken over to the search in the 2nd day. Figure 2 compares the evaluation task in the conventional IEC and the CEIEC As shown in example of CEIEC in Figure 2 (b), user's evaluation task is divided into fixed ten generations per one day, while user's evaluation task is finished in one day in the conventional IEC.

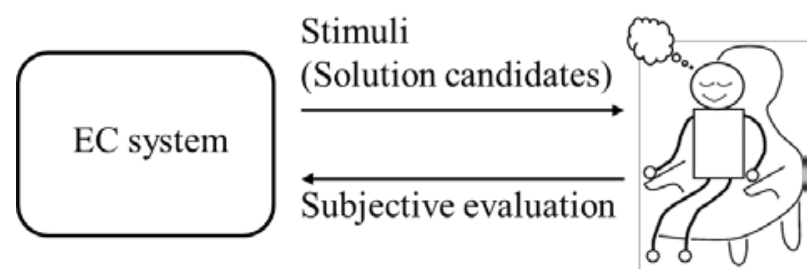

Figure 1: Typical relationship between system of Evolutionary Computation and human subject in Interactive Evolutionary Computation.

\section{(a) Conventional IEC}

\begin{tabular}{|l|l|l|l|l|l|}
\hline 1 & 2 & 3 & & $\cdots$ & $n-1 \mid n$ \\
\hline
\end{tabular}

(b) CEIEC

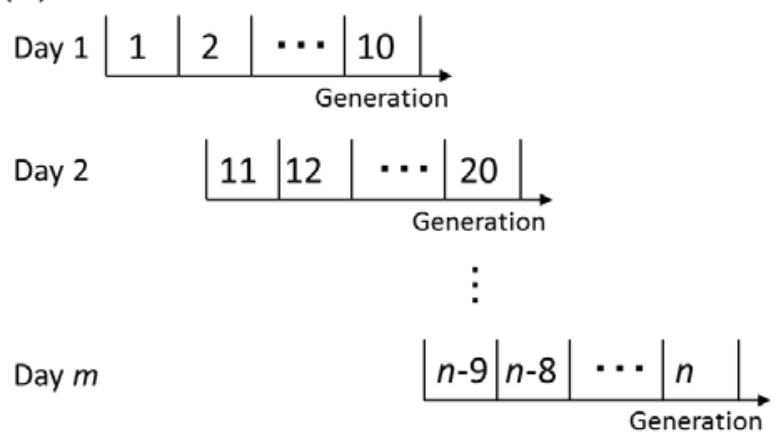

Figure 2: Comparison of evaluation task of user in IECs: (a) Conventional IEC, (b) Continuous Evaluation-based IEC.

\section{Interactive Evolutionary Computation system creating sign sound}

Most of previous IEC studies selected computer graphics and sound contents as the target of creation due to ease of operation in computer [2]. In this study, the efficiency of the proposed IEC is investigated by using an IEC system creating sign sounds. Some previous studies proposed IEC that searches sign sounds by employing Genetic Algorithm and Differential Evolution (DE) [9,10]

This study uses Interactive Differential Evolution (IDE) creating sign sounds [10]. IDE is an interactive type of DE which proceeds its search with a comparison of the current solution (target vector) and the new solution (trial vector) $[11,12]$. DE is known as an evolutionary algorithm having simple scheme, and a previous study investigated searching ability of DE and showed its superiority by comparing with conventional algorithm [13].

Fig. 3 shows how the IDE system creates sign sounds with music rules. The IDE system creating sign sounds represents a variety of short music melodies as sign sounds by using successive eight music notes. These music notes also play roles of extension of length of music note and rest note. In the previous study, the IDE successfully obtained user's "warning” sign sound throughout 13 generations [10].
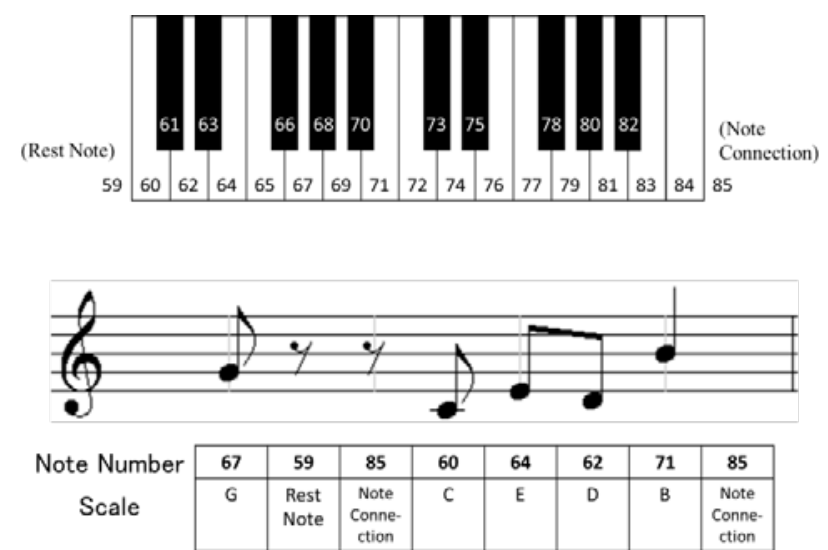

Figure 3: Correspondence between values in DE and its role in creation of sound contents [10].

\section{Listening Experiment}

Listening experiment was conducted for investigating the efficiency of the proposed IEC. The experiment was composed of two steps: a searching experiment and an evaluating experiment (Fig. 4). The target of search was a "bright" sign sound suited to each user's feeling. Sixteen persons individually participated in the experiment as subjects.

\subsection{Searching Experiment}

In this step, the subjects individually evaluated sounds created from the IDE system explained in the previous section. The evaluation was performed by pairwise comparison in IDE [14], and search in the IDE was progressed based on the evaluation. In the pairwise comparison, the subjects listened to two sign sounds sequentially and selected the brighter one. These two sign sounds were corresponded to the target and the trial vectors, and the sequence was randomized. 
The subjects performed the evaluation task through 30 generations which were divided into two days: they had the evaluation task through 15 generations in each of two days. The number of solution candidates (DE vectors) was eight in one generation. DE's parameters were set as $F=0.9$ and $C R=0.6$ respectively by referring to the previous study $[10,15,16]$.

\subsection{Evaluating Experiment}

In the evaluating experiment, three sign sounds were picked up from created population in the each user's searching experiment. The three sign sounds were subjectively evaluated its "brightness" with Semantic Differential method [17] in 7-point scale: 1-point meant extremely dark, 4-point meant neither, and 7-point meant extremely bright. The three sounds were randomly selected from 0th, 14th, and 29th generations, respectively. The subjects sequentially listened to the three sounds twice, and the order of presentation of the three sounds was randomized and counter-balanced.

\section{Experimental Results}

\subsection{Results of Searching Experiment}

Figure 5 shows the development of update of solution candidates during the searching experiment: these values are the mean and the standard deviation. The update of solution candidate means that replacement of the old solution (the target vector) with a new better solution (the trial vector) in DE algorithm. In the 1 st day (0-14 generations), gradual decrease in the number of updates was observed. It was changed from four times to two times. The number of update keeps around two times in the 2nd day (15-29 generations). No drastic changes between the numbers of the 14th and the 15th generations were observed.

Elapsed time for the evaluation tasks in the searching experiment was measured in each experimental day. Longer time needed in the evaluation was observed in the 1st day: fourteen out of sixteen subjects spent longer time in the 1st day. Mean time lengths were $2028.7 \mathrm{~s}$ in the $1 \mathrm{st}$ day and $1429.7 \mathrm{~s}$ in the $2 \mathrm{nd}$ day, respectively. With a sign test, the elapsed time in the 1 st day was significantly longer than in the 2 nd day $(P<0.01)$.

\subsection{Result of Evaluating Experiment}

Figure 6 shows the mean and the standard deviation of subjective fitness for the three selected sounds in the evaluating experiment. For the sign sounds of the 0th and 14th generations, almost same fitness around 4-point was obtained. The highest fitness was observed in the 29th generation. Almost same standard deviations were observed in all of the three generations.

For comparing these fitness values which were obtained in the different three generations, Shafer's multiple comparisons was employed. As a result of the analysis, signif- icant difference was observed only between the 0 th and the 29th generations $(P<0.05)$.

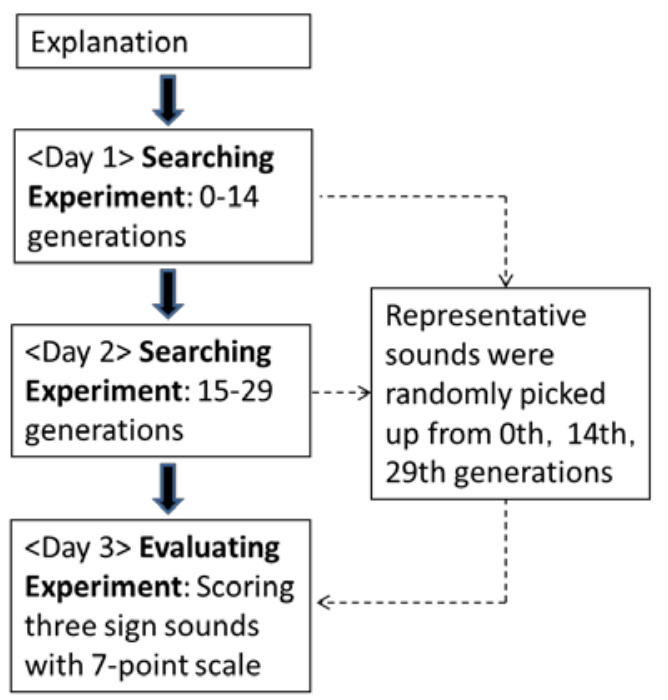

Figure 4: Experimental procedure composed of searching experiment and evaluating experiment.

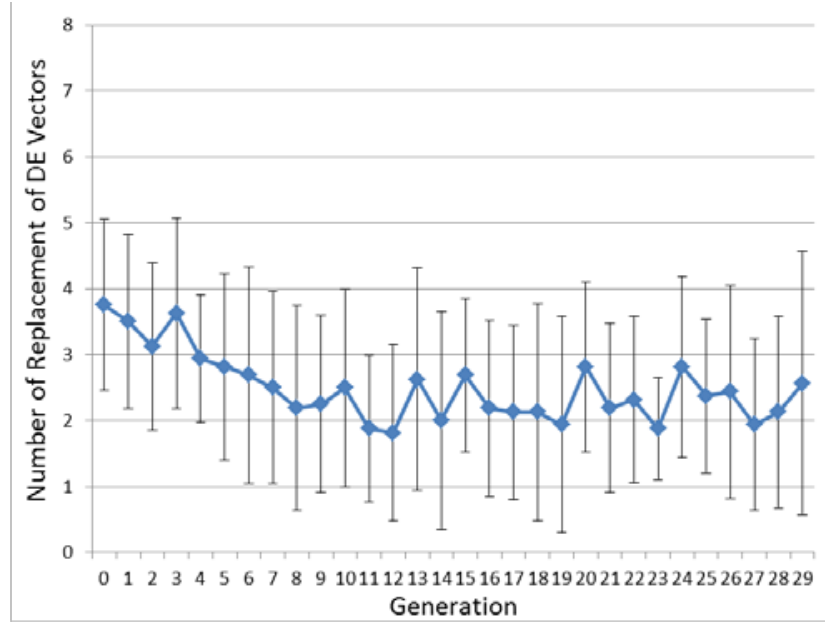

Figure 5: Progress of the number of replacement of DE vectors.

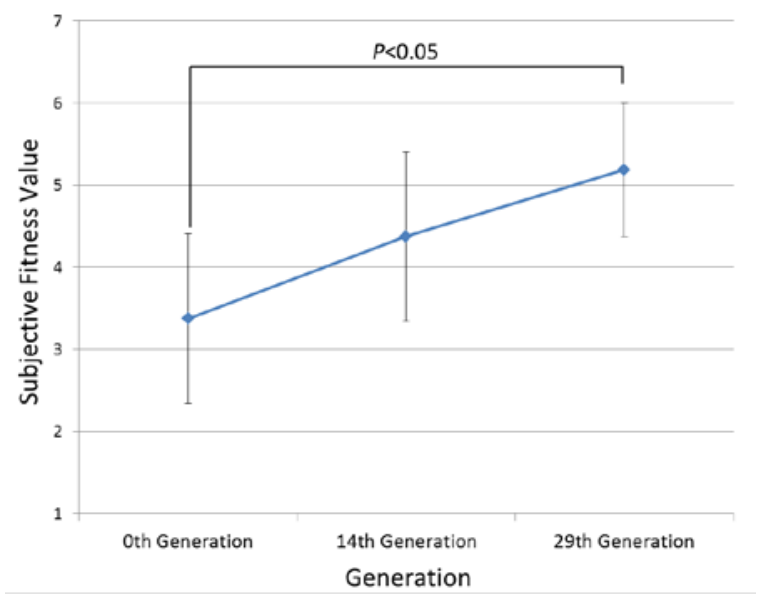

Figure 6: Progress of subjective fitness value for created sign sounds. 


\section{Discussion}

The gradual decrease in the number of update seems a trend of convergence in search which is observed in a general and successful search in EC. Continuous change in the number of updates between two days is considered as that the user's feeling for the sign sound is not largely different between two days.

Moreover, in the evaluating experiment, the solution having the highest fitness was found from the final generation in the 2 nd day. These results show a possibility of the CEIEC that enables the user to perform larger number of evaluations than conventional IECs. It means that the users will be able to obtain ideal media contents suited to each user's feelings over days.

Time length needed for evaluating 15 generations was significantly longer in the 1 st day than in the 2 nd day. This result means that the subjects were accustomed to the evaluation task selecting the brighter sign sound in the paired comparison. The same trend, decreasing time length for the evaluation task in accordance with generation, was observed in a previous IEC study related to creation of sound [18].

\section{Conclusion}

This study proposed a new concept of IEC with continuous evaluations. It divides user's evaluation tasks into different times and different days. Dividing the task is expected to contribute for reducing user's fatigue, since the subjects do not have to relatively long evaluation task through 30 generations in one day.

The efficiencies of the proposed IEC, CEIEC, were investigated through listening experiment composed of two steps. In the searching experiment which was divided into two days task, gradual decrease in the displacement of the old DE vector with the new DE vector was observed in the beginning of the search in the 1st day. After that, the replacement around two times was continuously observed. In the evaluating experiment, the highest fitness value was obtained in the sign sound picked up from the 29th generation. Result of the multiple comparisons showed significant difference between the 1st and the 29th generations. These results mean that the subject could obtain bright sign sound suited to his or her own feeling with CEIEC.

As future study, state of the convergence in the search process will be analyzed with a distance metrics between solution candidates of IDE in the search space. In the EC search, shrink of distance between solution candidates is observed when the search is successfully performed. Furthermore, we need to show efficiency of the proposed IEC through a statistical comparison in fitness values in the evaluating experiment with larger number of subjects. The performance of the search in the proposed IEC should be compared with the conventional IEC that performs the evaluation task in one day.

\section{References}

[1] R. Dawkins: The Blind Watchmaker, Penguin Books, USA, 1986

[2] H. Takagi: Interactive Evolutionary Computation: Fusion of the Capabilities of EC Optimization and Human Evaluation, Proc. the IEEE, 89(9), pp.1275-1296, 2001.

[3] H. Nishino, K. Takekata, M. Sakamoto, B. A. Salzman, T. Kagawa, and K. Utsumiya: An IEC-Based Haptic Rendering Optimizer, in Proc. IEEE WSTST'05, pp.653-662, 2005.

[4] K. Takekata, B. A. Salzman, H. Nishino, T. Kagawa, and K. Utsumiya: An Intuitive Optimization Method of Haptic Rendering Using Interactive Evolutionary Computation, in Proc. the IEEE Int. Conf. Systems, Man, \& Cybernetics, pp.1896-1901, 2005.

[5] A. A. G. Dharma, H. Takagi, and K. Tomimatsu: Emotional Expressions of Vibrotactile Haptic Message Designed by Paired Comparison-based Interactive Differential Evolution, in Proc. Evolutionary Computation Symposium 2011, S4-01, 2011 (in Japanese).

[6] M. Fukumoto and T. Ienaga: A Proposal for Optimization Method of Vibration Pattern of Mobile Device with Interactive Genetic Algorithm, in Proc. HCII, 11, pp.264-269, 2013.

[7] M. Fukumoto and J. Imai: Design of Scents Suited with User's Kansei using Interactive Evolutionary Computation, in Proc. Int. Conf. on Kansei Engineering and Emotion Research 2010, pp.1016-1022, 2010.

[8] M. Fukumoto, S. Koga, M. Inoue, and J. Imai: Interactive Differential Evolution Using Time Information Required for User's Selection: In A Case of Optimizing Fragrance Composition, in Proc. 2015 IEEE Congress on Evolutionary Computation, pp.2192-2198, 2015.

[9] M. Miki, H. Orita, S. H. Wake, and T. Hiroyasu: Design of Sign Sounds using an Interactive Genetic Algorithm, in Proc. the IEEE Int. Conf. Systems, Man, \& Cybernetics 2006, 4, pp.3486-3490, 2006.

[10] M. Fukumoto: An Efficiency of Interactive Differential Evolution for Optimization of Warning Sound with Reflecting Individual Preference, IEEJ Transactions on Electrical and Electronic Engineering, 10(S1), pp.S77-S82, 2015.

[11] R. Storn and K. V. Price: Differential evolution-A simple and efficient adaptive scheme for global optimization over continuous spaces, Institute of Company Secretaries of India, Chennai, Tamil Nadu. Tech. Report TR-95-012, 1995.

[12] K. V. Price, R. Storn, and J. Lampinen: Differential Evolution: A Practical Approach to Global Optimization, Springer-Verlag, Germany, 2005.

[13] J. Vesterstrøm and R. Thomson: A comparative study of differential evolution, particle swarm optimization, and evolutionary algorithms on numerical benchmark problems, in Proc. 2004 IEEE Congress on Evolu- 
tionary Computation, 2, pp.1980-1987, 2004.

[14] H. Takagi and D. Pallez: Paired Comparison-based Interactive Differential Evolution, in Proc. World Congress on Nature and Biologically Inspired Computing, pp.375-380, 2009.

[15] R. Gamperle, S. D. Muller, and A. Koumoutsakos: Parameter study for differential evolution, in Proc. WSEAS NNA-FSFS-EC 2002, pp.293-298, 2002.

[16] J. Ronkkonen, S. Kukkonen, and K. V. Price: Real-parameter optimization with differential evolution, in Proc. 2005 IEEE Congress on Evolutionary Computation, 1, pp.506-513, 2005.

[17] C. E. Osgood, G. K. Suci, and P. Tannenbaum: The measurement of meaning, University of Illinois Press, USA, 1957.

[18] M. Fukumoto, R. Yamamoto, and S. Ogawa: The Efficiency of Interactive Differential Evolution in Creation of Sound Contents: In Comparison with Interactive Genetic Algorithm, International Journal of Software Innovation, 1-2, pp.16-27, 2013. 